\title{
A Survey of Microaneurysms Detection using Segmentation Techniques in Fundus Images
}

\author{
Priyanka Powar \\ Student \\ DYPIET, Pimpri, Pune
}

\author{
C. R. Jadhav \\ Assistant Professor \\ DYPIET, Pimpri, Pune
}

\begin{abstract}
Diabetic Retinopathy is a major medical problem that causes damage to the eye. A need arises to detect it at an early stage. Since this ailment is symptomless, it can only be a diagnosed by oculist. Currently, the trained eye care specialists are not able to screen the exponential increase in the number of Diabetic Retinopathy patients. An automated Diabetic Retinopathy screening system will enable the detection of lesions accurately, thus helping the ophthalmologists. Microaneurysms are the earliest clinical signs of Diabetic Retinopathy. They are reddish in color and appear as small red spots on the retinal fundus images. Early detection of microaneurysm can help in the early treatment of Diabetic Retinopathy. This paper presents the study and review of various techniques used in detection of microaneurysm from the diabetic retinopathy images. This paper is motivated by need of increasing sensitivity and reducing computational time for detection and classification of microaneurysm from the diabetic retinopathy images. Retina images are obtained from the fundus camera and graded by skilled professionals. However there is considerable shortage of expert observers has encouraged computer assisted monitoring. Evaluation of blood vessels network plays an important task in a variety of medical diagnosis. Manifestations of numerous vascular disorders, such as diabetic retinopathy, depend on detection of the blood vessels network.In this work the fundus RGB image is used for obtaining the traces of blood vessels and areas of blood vessels are used for detection of Diabetic Retinopathy (DR).
\end{abstract}

\section{Keywords}

Diabetic Retinopathy, Fundus image, Microaneurysm Detection, Retinal Image, Screening.

\section{INTRODUCTION}

Diabetes has turn out to be one of the quickly increasing diseases worldwide. Diabetic Retinopathy is one of the leading causes of blindness. Usually there are no early visible symptoms of the Diabetic Retinopathy(DR) and as the disease progresses the presence of micro aneurysms (MAs), exudates both hard and soft and new blood vessels can be observed. Effective diagnosis of Diabetic Retinopathy and treatment can prevent blindness. Due to the large number of people that require screening, an automated and accurate screening tool is a useful adjunct in diabetes clinics. Accordingly, data stream mining method should react to those changes.

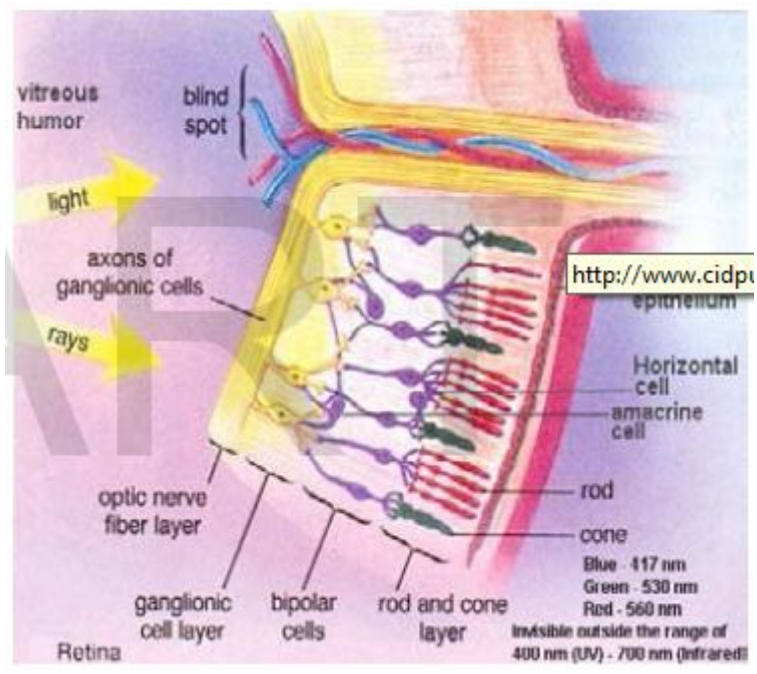

Fig.1 Retinal Structure [12]

As the disease is treatable, detection and monitoring of the disease via fundus photography is beneficial and more efficient detection and monitoring saves costs. It would seem that automated image detection of diabetic retinopathy is an engineering solution to an increasing need. Fundus imaging has an important role in diabetes monitoring since occurrences of retinal abnormalities are common and their consequences serious. However, since the eye fundus is sensitive to vascular diseases, fundus imaging is also considered as a candidate for non-invasive screening. The success of this type of screening approach depends on accurate fundus image capture, and especially on accurate and reliable image processing algorithms for detecting the abnormalities. In this paper, a new technique, method and algorithm surveyed for efficient detection of micro aneurysms from fundus images. The first signs of DR are Microaneurysms (MAs), tiny dilations of the capillaries that do not affect vision. Moreover, MAs detection is the most important step in the automated detection of DR systems. MAs are visible immediately after the arterial phase of fluoresce in angiography (FA), a technique used to evaluate the circulation in the retina and choroid. MAs counting have been used as a tool for determination of the progression of the DR and there are many protocols that perform this task. A number of different methods for automated MA detection have been proposed in the past Nevertheless, these methods were tested on different databases using various evaluation measures that made it impossible to direct comparison of performance [14].

\section{RELATED WORK}

Spencer Timothy and john Olson [1] describes different segmentation techniques which detects and quantifies microaneurysms present in digitized fluorescein angiograms. Microaneurysms were segmented by using bilinear top-hat 
transformation and matched filtering technique. A novel region-growing algorithm fully delineates each marked object and subsequent analysis of the size, shape, and energy characteristics as well as fully grown objects accumulated in separate binary image. This strategy is valuable in providing a way of accurately monitoring the progression of diabetic retinopathy.

Niemeijer, Meindert, et al. [2] demonstrated Hybrid approach used to detection of red lesions in digital color fundus. Proposed a Pixel classification method which separates vasculature and red lesions from background of the image. Also K-nearest neighbour classifier used to classify detected objects.

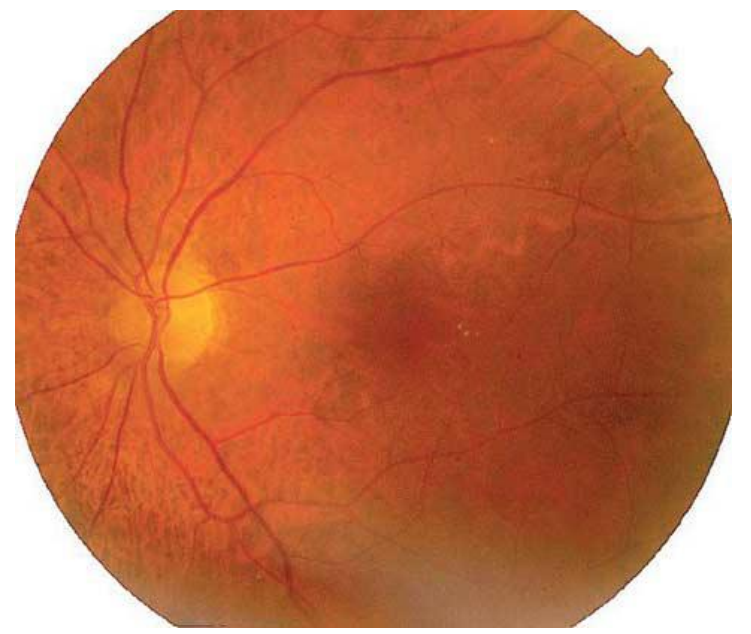

Fig.2 A digital color fundus photograph containing red lesions [2].

Fleming, Alan D., et al. Fleming, Alan D., et al. [3] described that image contrast normalization can improve the ability to distinguish between MAs and other dots that occur on the retina. Different methods compared for contrast normalization. Watershed transform method gave best results to derive a region that contains no vessels or other lesions. Local vessel detection technique handled successfully dots within vessels.

Walter, Thomas, et al. [4] proposed new algorithm which divides into four stages for automatic detection of MA's in color fundus images. In first step, image enhancement, shade correction and image normalization. Second stage designed for detection of candidate that all patterns originated to MA which achieved by diameter closing and automatic threshold scheme. In last stage, feature extracted to automatically classify candidate into real MA and other objects.

Quellec, Gwénolé, et al. [5] proposed an automatic method to detect microaneurysms in retina photographs. Microaneurysms are the most frequent and usually the first lesions to appear as a consequence of diabetic retinopathy. So, their detection is necessary for both screening the pathology and follow up. Automating this task, which is currently performed manually, would bring more objectivity and reproducibility. So, to detect them by locally matching a lesion template in sub bands of wavelet transformed images. To improve the method performance, was searched for the best adapted wavelet within the lifting scheme framework. The optimization process is based on a genetic algorithm followed by Powell's direction set descent. Results are evaluated on 120 retinal images analyzed by an expert and the optimal wavelet is compared to different conventional mother wavelets. These images are of three different modalities: there are color photographs, green filtered photographs, and angiographs. Depending on the imaging modality, microaneurysms were detected with sensitivity.

Esmaeili, Mahdad, et al. [6] demonstrated new curvelet based algorithm to separate red lesions from rest retinal image. Also to improve non-uniform background introduced new illumination equalizations algorithm. This prevent from the creating dark shade around light lesions. In next stage, applied digital curvelet transform to produced enhanced images and to modified curvelet coefficient in order to lead red objects to zero and also gives sparse representation of the object.

Sopharak, Akara, Bunyarit Uyyanonvara, and Sarah Barman[13]introduced Various segmentation techniques were used to detect tiny sizes, low contrast, and similar blood vessels of microaneurysms. There used two segmentation techniques, one coarse segmentation using mathematical morphology which to identify MA candidate in retinal images and fine segmentation using naïve bayes classifier which mainly in areas of computer vision and image processing. Bayesian network an important formalism for medical decision support system.

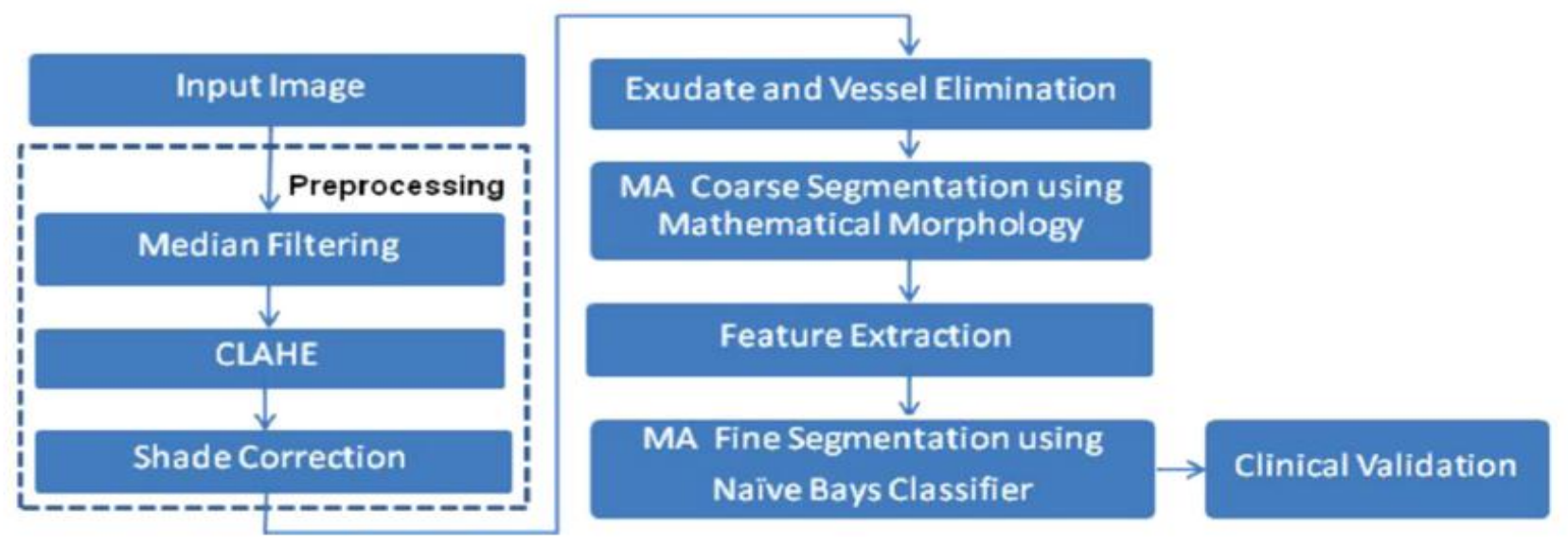

Fig.3 Procedure of microaneurysms detection[12]

Adal, Kedir M., et al. [15] designed Microaneurysm detection problem as finding interest region or blobs from image. Characterized these blob regions by scale adapted region descriptor. Semi-Supervised based learning approach proposed to train a classifier which can detect true MAs. A Gaussian mixture-model based clustering combined 
andlogistic regression classification method has been proposed toclassify microaneurysms at pixel level.

Antal, Bálint, and András Hajdu. [10] Presented two approaches to improve Microaneurysm detection methods. In first, selects set of preprocessing methods for candidate extractor and it measure in six different MA categories and for each different category best preprocessing method selected and organized into an ensemble based method. Second, adaptive weighting approach presented which actual works on spatial locations were categorized into: near to vessel, in the macula, on the periphery and also works on contrast of the detected microaneurysms.

labels which are continuous as well as hierarchical, author proposed hierarchical continuous label classifier algorithm(HCC) to construct decision tree. Discretization is performed dynamically during tree construction. This proposed approach has standard structure of previous methods such as C4.5 and ID3. Tavakoli, Meysam, et al. [14] proposed a novel method based on random transform (RT) and multioverlapping for early detection of microaneurysms. Initially, optic nerve head $(\mathrm{ONH})$ was detected or masked and to remove the background top-hat transformation and averaging filter were applied in preprocessing stages. After detecting and masking retinal vessels and $\mathrm{ONH}$, microaneurysms were detected and numbered by using RT and thresholding.

\section{CONCLUSION}

This paper described survey of previous researches for micro aneurysm detection, advantages and disadvantages of existing method. Also present the various segmentation techniques of microaneurysms detection. As per survey, there is strong need of reducing computational time and increasing sensitivity to detect and classify microaneurysms. Surveyed some methods that are evaluating and comparing with automatic detection of diabetic retinopathy.

\section{REFERENCES}

[1] Spencer, Timothy, et al. "An image-processing strategy for the segmentation and quantification of microaneurysms in fluorescein angiograms of the ocular fundus." Computers and biomedical research 29.4 (1996): 284-302.

[2] Niemeijer, Meindert, et al. "Automatic detection of red lesions in digital color fundus photographs." Medical Imaging, IEEE Transactions on 24.5 (2005): 584-592.

[3] Fleming, Alan D., et al. "Automated microaneurysm detection using local contrast normalization and local vessel detection." Medical Imaging, IEEE Transactions on 25.9 (2006): 1223-1232.
[4] Walter, Thomas, et al. "Automatic detection of microaneurysms in color fundus images." Medical image analysis 11.6 (2007): 555-566.

[5] Quellec, Gwénolé, et al. "Optimal wavelet transform for the detection of microaneurysms in retina photographs." Medical Imaging, IEEE Transactions on 27.9 (2008): 1230-1241

[6] Esmaeili, Mahdad, et al. "A new curvelet transform based method for extraction of red lesions in digital color retinal images." Image Processing (ICIP), 2010 17th IEEE International Conference on. IEEE, 2010.

[7] Fadzil, MH Ahmad, Lila Iznita Izhar, and Hanung Adi Nugroho. "Determination of foveal avascular zone in diabetic retinopathy digital fundus images."Computers in biology and medicine 40.7 (2010): 657-664

[8] Niemeijer, Meindert, et al. "Retinopathy online challenge: automatic detection of microaneurysms in digital color fundus photographs." Medical Imaging, IEEE Transactions on 29.1 (2010): 185-195.

[9] Bae, Jang Pyo, et al. "A study on hemorrhage detection using hybrid method in fundus images." Journal of digital imaging 24.3 (2011): 394-404.

[10] Antal, Bálint, and András Hajdu. "Improving microaneurysm detection in color fundus images by using context-aware approaches." Computerized Medical Imaging and Graphics 37.5 (2013): 403-408.

[11] Akram, M. Usman, Shehzad Khalid, and Shoab A. Khan. "Identification and classification of microaneurysms for early detection of diabetic retinopathy."Pattern Recognition 46.1 (2013): 107-116.

[12] Kade Mahesh k, "A Survey of Automated Techniques for Retinal Disease Identification in Diabetic Retinopathy". International Journal of Advancements in Research \& Technology, May-2013 ISSN 2278-7763.

[13] Sopharak, Akara, Bunyarit Uyyanonvara, and Sarah Barman. "Simple hybrid method for fine microaneurysm detection from non-dilated diabetic retinopathy retinal images." Computerized Medical Imaging and Graphics 37.5 (2013): 394-402

[14] Tavakoli, Meysam, et al. "A complementary method for automated detection of microaneurysms in fluorescein angiography fundus images to assess diabetic retinopathy." Pattern Recognition 46.10 (2013): 2740 2753.

[15] Adal, Kedir M., et al. "Automated detection of microaneurysms using scale-adapted blob analysis and semi-supervised learning." Computer methods and programs in biomedicine 114.1 (2014): 1-10. 